\title{
New Species of the Genus Psylliodes Latr. (Coleoptera, Chrysomelidae) from the Palaearctic Region
}

\author{
K. S. Nadein \\ Zoological Institute, Russian Academy of Sciences, St. Petersburg, 199034 Russia \\ Received June 29, 2006
}

\begin{abstract}
Six new species of the genus Psylliodes Latr. are described from the Palaearctic Region. P. concolor $\mathrm{sp} . \mathrm{n}$. from Georgia and P. diversicolor $\mathrm{sp}$. $\mathrm{n}$. from Turkey belong to the luteolus species-group. P. amurensis $\mathrm{sp} . \mathrm{n}$. and $P$. laxus sp. $\mathrm{n}$. from the Russian Far East are included in the punctifrons species-group. P. submontanus sp. $\mathrm{n}$. from the North Caucasus is provisionally included in the napi species-group, but it is similar to P. ozisiki Leon. et Arnold from the cupreus species-group. P. insularis sp. $\mathrm{n}$. from the Canaries belongs to the chrysocephalus species-group.
\end{abstract}

DOI: $10.1134 / \mathrm{S} 0013873806080094$

As the result of examination of a material on the large genus Psylliodes Latr. from the Palaearctic Region, deposited in the collection of the Zoological Institute, Russian Academy of Sciences (St. Petersburg), six new species of the genus were revealed, their descriptions are given below.

Type specimens of the all species described are deposited in the Zoological Institute, Russian Academy of Sciences, St. Petersburg.

The following abbreviations are used in the paper: PI, pronotum length to width ratio; EI, elytra length to width ratio; LI, pronotum to elytra length ratio; WI, pronotum to elytra width ratio; BI, body length to width ratio.

\section{Psylliodes amurensis Nadein, sp. n.}

Material. Holotype: $\widehat{\jmath}$, "promysel Ozerpakh, liman of Amur, Chernavin, 3.VIII.915."

Description. Body oblong-oval, not wide, moderately convex, dorsally nearly black, strongly shining, with weak bronze shine; head and elytral apex paler; antenna, fore and middle legs, and hind tibia rufousyellow, bases of fore and middle femora slightly darker, hind femur brown, darker in upper part; ventrally body dark brown.

Head not large, not elongate; eyes very large and strongly convex. Vertex moderately wide, weakly convex, shining, punctate between eyes; punctures uneven, variable in size and depth, irregularly arranged; surface of vertex between punctures bearing small tubercles. Ocular sulcus rather narrow and rather deep, with even smooth margins, adjoining margin of eye along nearly entire length, separated from it by short interval only near frontal calli; large setiferous pore, forming no depression, situated at inner upper margin of eyes near sulcus. Frontal calli poorly developed, indistinct, not convex, smooth, not separated from vertex by lines, more distinctly separated from frons. Frontal ridge narrow, triangular, short, weakly convex, with smooth upper surface and flat base; anterofrontal ridge narrow and weakly convex, weakly emarginate in middle. Antennal grooves rather shallowly depressed, slightly tuberculate, shagreened. Gena very short. Labrum moderately wide and short; setiferous pores small and superficial.

Pronotum slightly wider than long; lateral margins slightly converging forwards, weakly rounded; edging of anterior margin almost invisible, that of posterior margin well developed, convex, fine; edgings of lateral margins narrow, smooth; fine superficial depression, interrupted in middle, running along basal margin of pronotum. Anterolateral callosity moderately developed, not thickened, narrow, weakly projecting beyond contour, forming distinct rounded angular projection near setiferous pore. Punctation of disc moderately dense, denser at sides; punctures arranged not quite regularly, separated by intervals, which on disc, on average, 1.5-2.5 times puncture diameter; punctures occasionally irregular-shaped, moderately deep, usually small, but occasionally varying in size. Surface between punctures more or less smooth or not quite even, with inconspicuous smoothened shagreen- 

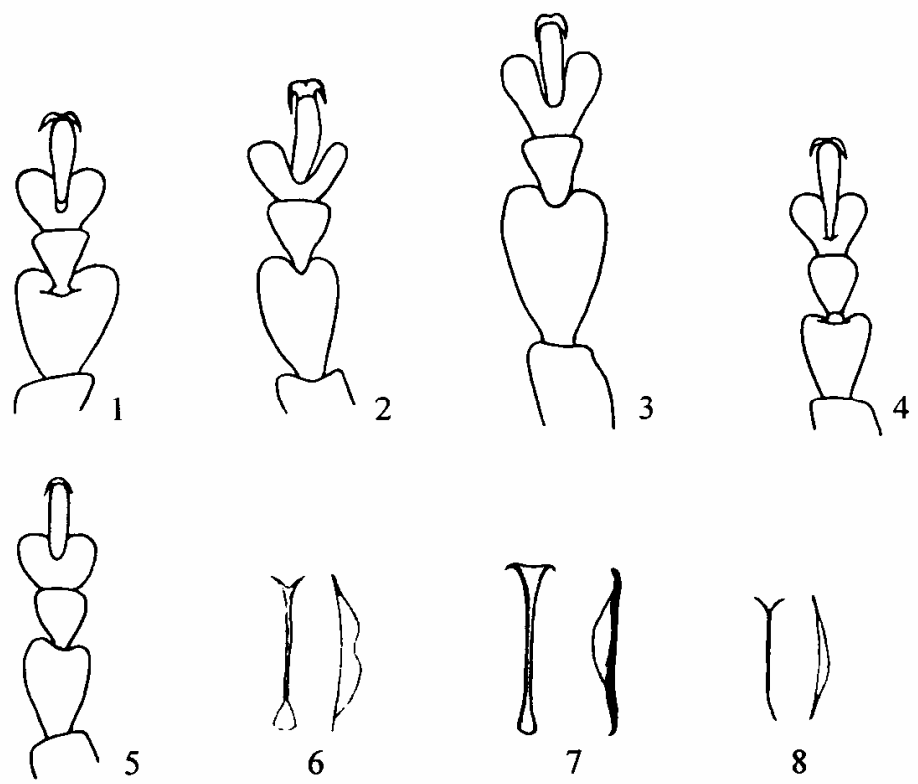

Figs. 1-8. Psylliodes Latr.: (1-5) fore tarsus, male [(1) P. diversicolor sp. n., (2) P. amurensis sp. n., (3) P. laxus sp. n., (4) P. concolor sp. n., (5) P. submontanus sp. n.]; (6-8) tegmen, dorsal and lateral view [(6) P. diversicolor sp. n., (7) P. concolor sp. n., (8) P. submontanus sp. n.].

ity-like microsculpture visible only under great magnification.

Scutellum paler than pronotum and elytra, triangular, with rounded apex and very finely shagreened surface. Elytra elongate, with lateral margins subparallel in middle. Humeral calli well developed, rather convex. Punctate striae not forming depressed sulci, intervals between striae flat or weakly convex. Strial punctures large, deep, larger and deeper than those on pronotum; intervals between punctures in striae not exceeding half puncture diameter, frequently shorter; outer stria deeply depressed, punctures arranged very densely, nearly merging; on disc, intervals between striae 1.5-2.0 times puncture diameter; striae nearly reaching elytral apex; punctures at apex nearly as large as at base; intervals between striae with rather delicate and superficial secondary punctation, punctures arranged moderately densely, occasionally forming one confused stria; intervals between punctures uneven, occasionally with very weak wrinkles and strokes. Apices of elytra separately distinctly rounded toward suture; sutural angle strongly rounded.

Abdominal sternite $\mathrm{V}$ of male with large deep rounded depression. Hind tibia narrow, moderately curved along lower margin, inner ridge with large apical tooth; apex in dorsal view slightly widened, with emargination in middle of inner margin (Fig. 10).
First segment of fore tarsus of male large and moderately wide (Fig. 2). First segment of hind tarsus straight. Tegmen modified. Aedeagus as in Fig. 16.

Length of body $2.56 \mathrm{~mm}$, width $1.26 \mathrm{~mm}$; PI 1.40, EI 1.56, LI 3.26, BI 2.03, WI 1.50.

Differential diagnosis. $P$. amurensis is closely related to P. subrugosus Jac. and differs from it in the following characters: head in area of frontal calli less strongly convex; labrum longer and narrower; punctation of vertex irregular, formed by punctures varying in size and separated by uneven intervals; punctures on pronotum more distinct, deeper, and separated by smoother intervals (occasionally shagreened); punctures in elytral striae denser; secondary punctation coarser; 1st segment of fore tarsus of male less strongly widened; body dorsally, legs, and antennae colored otherwise; and aedeagus shorter and wider, straight in lateral view.

This species belongs to the punctifrons speciesgroup also including $P$. cyanescens $\mathrm{W}$ se., $P$. subrugosus Jac., and P. laxus sp. n.

Distribution. Russia: the Far East.

Psylliodes laxus Nadein, sp. $\mathrm{n}$.

Material. Holotype: $\partial^{\lambda}$, "Ussuri Basin, Bikin. Distr., Birskoe, 4.7.1958, Kabakov." Paratypes: "Ev- 


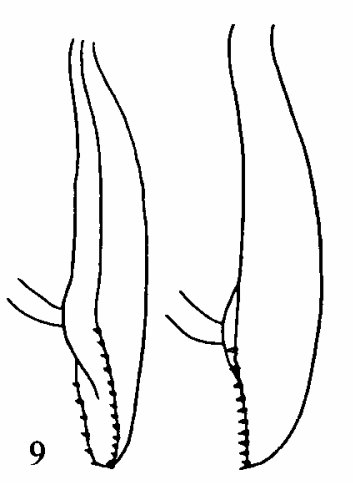

10

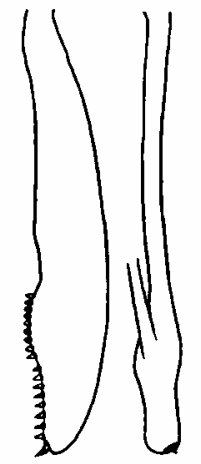

11
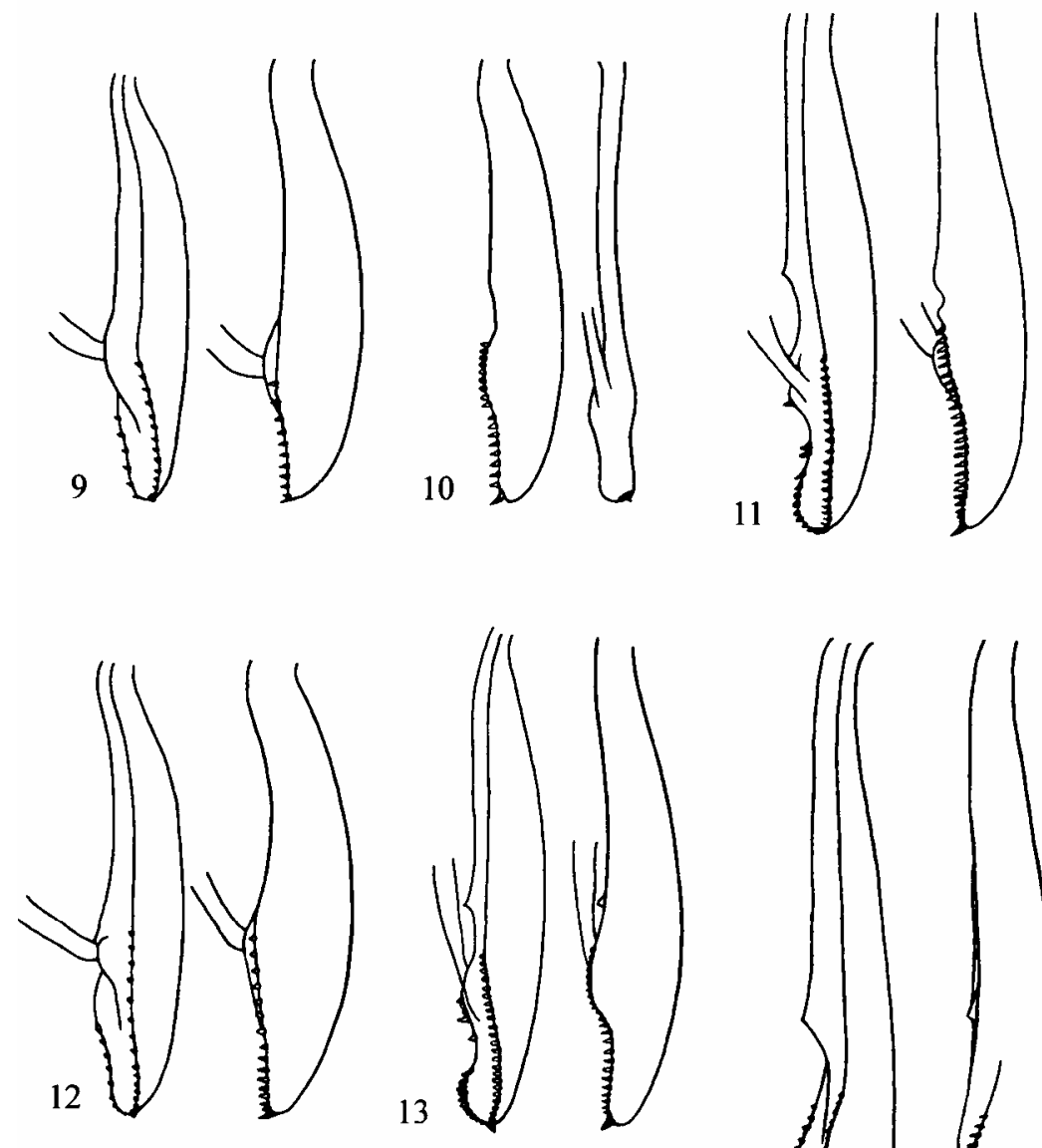

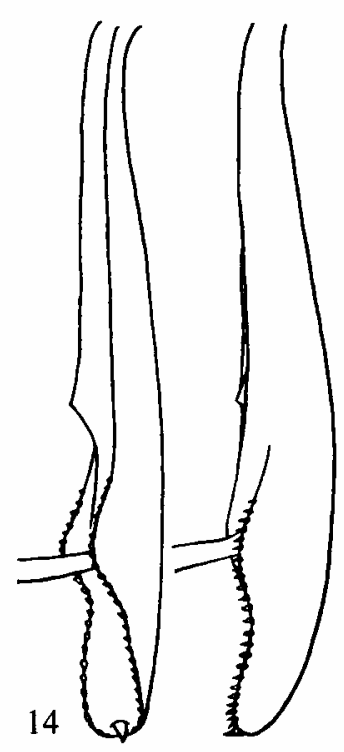

Figs. 9-14. Psylliodes Latr., hind tibia, dorsal and lateral view: (9) P. diversicolor sp. n., (10) P amurensis sp. n., (11) P. laxus sp. n., (12) P. concolor sp. n., (13) P. submontanus sp. n., (14) P. insularis sp. n.

seevka, Yuzhno-Ussuriiskii Terr., N. Titarev, 78.V.910," 1 +; "Pravda, S of Kholmsk, southern Sakhalin, Kerzhner, 26.V.973, 1 \%.

Description. Body oblong-oval, moderately convex, dorsally black with silky shine to dark greenish blue with metallic silky shine; antenna, fore and middle legs, and hind tibia yellowish rufous; apical antennal segments and bases of fore and middle femora slightly darker; hind femur from brownish with dark apex to black.

Head small; eyes moderately large, convex. Vertex nearly flat in lateral view, entirely covered with distinct deep punctures as large as, or slightly smaller than those on pronotum; intervals between punctures occasionally equal to diameter of punctures (more frequently shorter), flat or slightly convex, with distinct coarse granulate shagreenity. Ocular sulcus reaching antennal socket, deep, with even margins; at inner upper margin of eye, sulcus wider, with less steeply sloping margin from side of vertex, margin and bottom of sulcus with several small, and one large setiferous pores; near frontal calli, sulcus narrower, separated from eye by narrow interval; margin from side of vertex even and slightly ledging. Frontal calli narrow, not separated from vertex, nearly flat, smooth, distinctly separated from frons. Frontal ridge rather wide and triangular to narrow and subparallel-sided, moderately convex, shagreened; anterofrontal ridge weakly convex, narrow, straight. Antennal groove moderately deep, tuberculate, and coarsely shagreened. Labrum moderately large, not very 

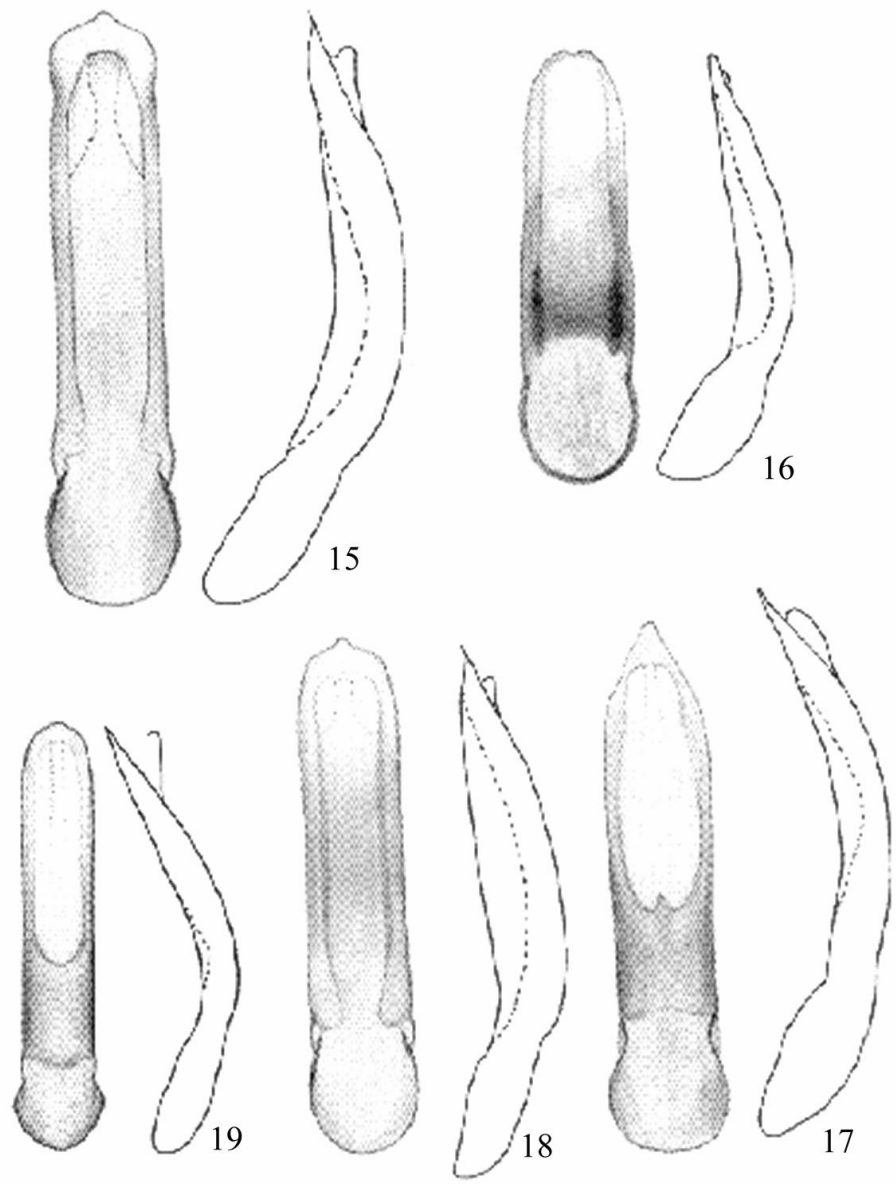

Figs. 15-19. Psylliodes Latr., aedeagus, ventral and lateral view: (15) P. diversicolor $\mathrm{sp} . \mathrm{n}$. , (16) P. amurensis $\mathrm{sp} . \mathrm{n}$., (17) P. laxus $\mathrm{sp}$. n., (18) P. concolor sp. n., (19) P. submontanus sp. n.

long; setiferous pores developed, especially two inner ones, large and deep, situated closely to one another.

Pronotum slightly wider than long, moderately convex. Sides weakly converging forwards, slightly rounded; edging of anterior margin very fine, hardly visible, that of posterior margin wider, convex; edgings of lateral margins narrow, smooth; anterolateral callosity well developed, weakly thickened, distinctly, but not very strongly projecting beyond contour, with distinct angular projection near setiferous pore; punctation large, punctures smaller than those on elytra, distinct, deep, dense; intervals between punctures not convex, covered with distinct coarse reticulate-granulate shagreenity, their average length 1.0-1.5 times puncture diameter.

Scutellum triangular, weakly rounded at apex, nearly sharp, convex, with smoothened shagreenity. Elytra elongate, moderately convex, with very weakly rounded sides; humeral calli moderately large, moder- ately convex; strial punctures small, as large as, or slightly larger than those on pronotum, superficial, distinct, frequently slightly elongate; striae occasionally slightly uneven, not forming depressed sulci; intervals between striae flat, 2.5-4.5 times puncture diameter, their average length nearly 3 times puncture diameter; intervals between punctures in striae about 0.5-1.0 times puncture diameter; punctures at bases of elytra larger and deeper than those at apices; secondary punctation rather fine, superficial, hardly visible against background of shagreenity, sparse, occasionally nearly absent; intervals between punctures with very fine or distinct, granulate or slightly flattened shagreenity. Epipleura with uneven tuberculatewrinkled sculpture.

Hind tibia long and wide, straight, weakly or moderately widened in apical $1 / 3$ in dorsal view; inner ridge with 1 large tooth, outer ridge occasionally bearing several short denticles-notches (Fig. 11). First segment of hind tarsus straight. First segment of fore 

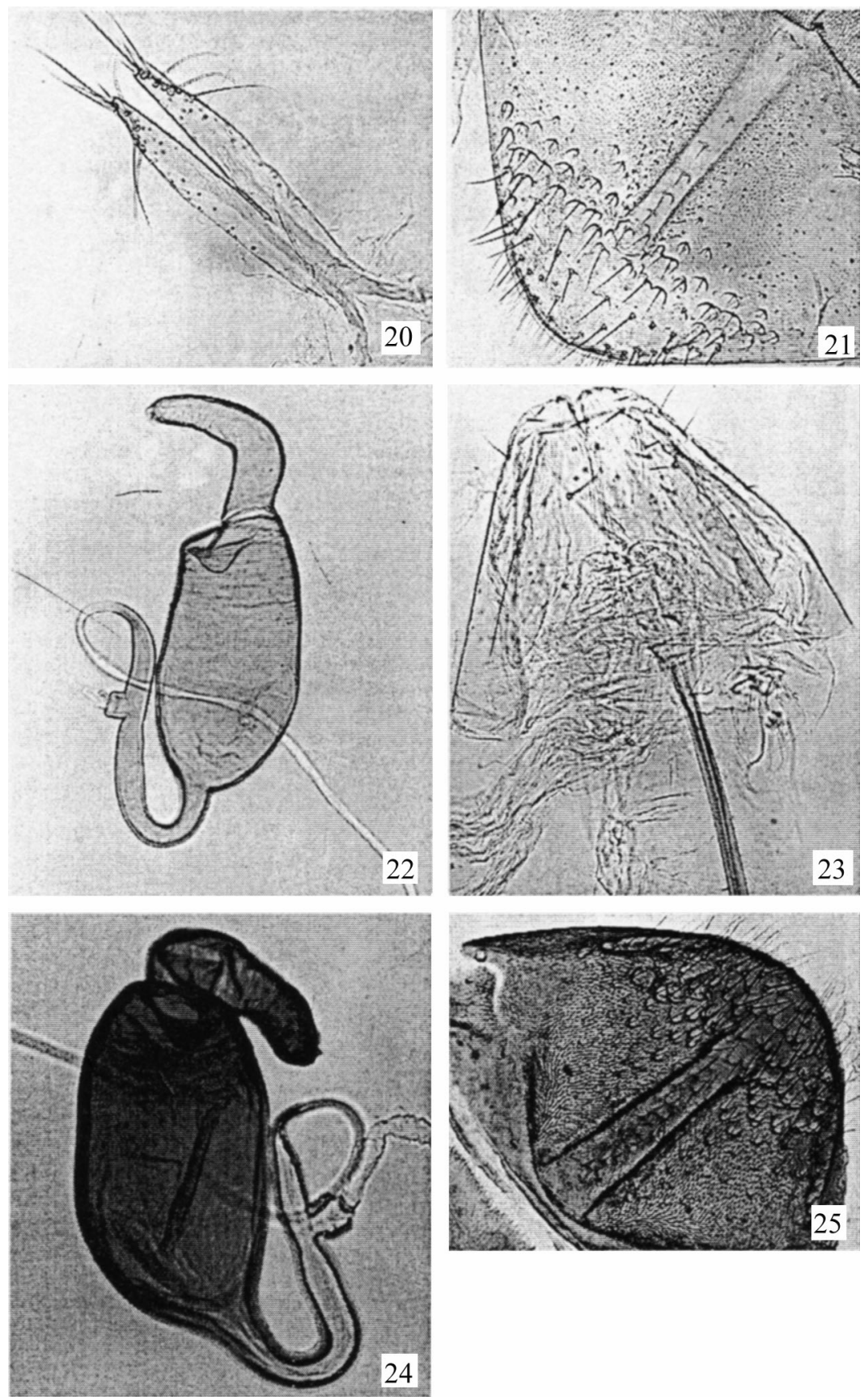

Figs. 20-25. Psylliodes Latr.: (20-23) P. insularis sp. n. [(20) vaginal palpi, (21) pygidium, (22) spermatheca, (23) spiculum ventrale], $(24,25)$ P. laxus sp. n. [(24) spermatheca, (25) pygidium].

tarsus of male very large and wide (Fig. 3). Tegmen modified. Aedeagus as in Fig. 17.

Female. Spermatheca with short and thick collo; nodulus medium-sized, moderately wide; ductus short, not curved and not forming loops (Fig. 24). Pygidium as in Fig. 25, vaginal palpi as in Fig. 26, spiculum ventrale as in Fig. 27.
Length of body of holotype $2.97 \mathrm{~mm}$, width $1.54 \mathrm{~mm}$; those of paratypes 3.26 and $1.48-1.54 \mathrm{~mm}$, respectively; PI 1.38-1.42 (1.40), EI 1.47-1.70 (1.60), LI 3.24-3.40 (3.34), BI 1.93-2.20 (2.08), WI 1.41$1.59(1.50)$.

Differential diagnosis. The new species is very closely related to P. takizawai Gruev and clearly dif- 

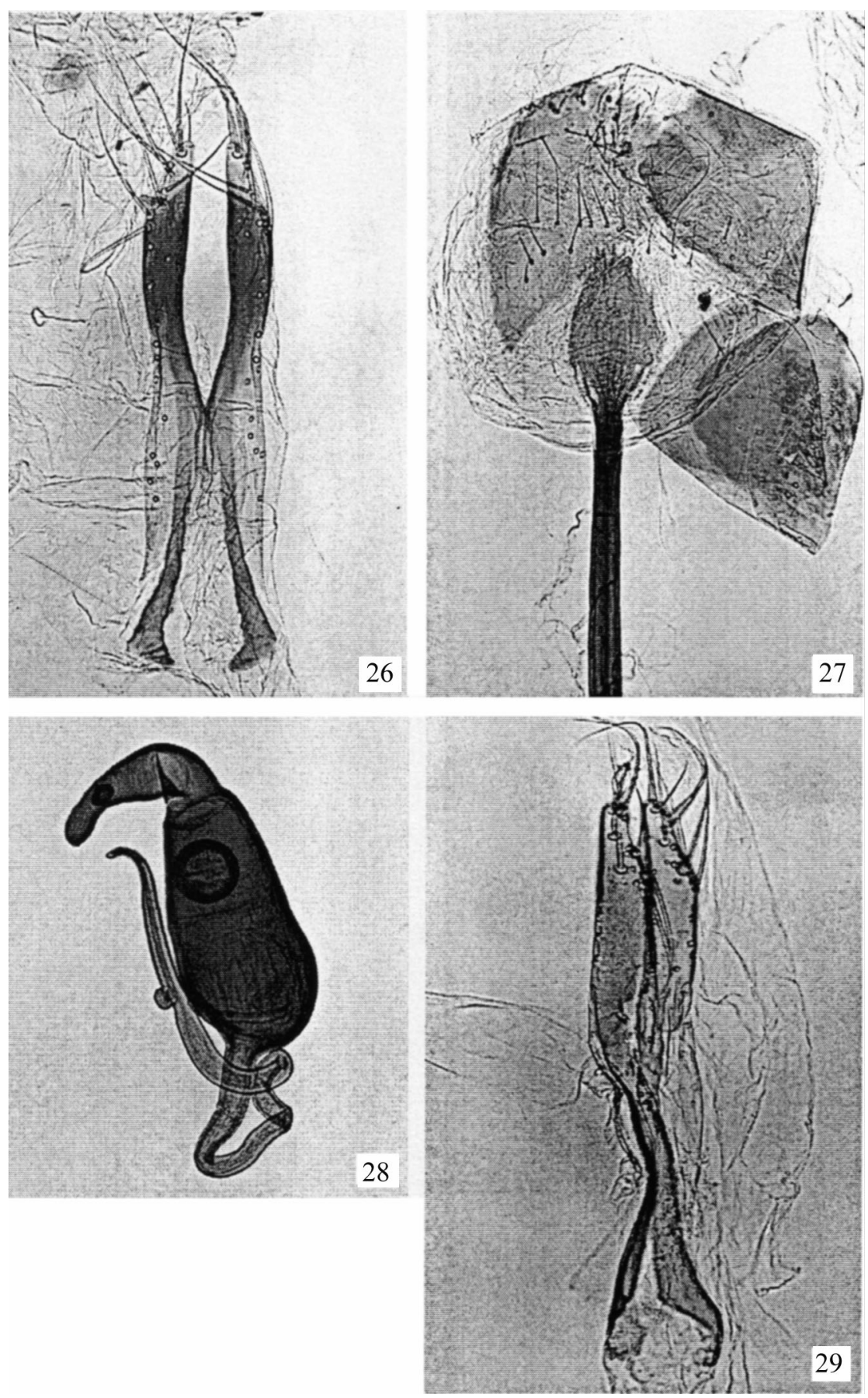

Figs. 26-29. Psylliodes Latr.: (26, 27) P. laxus sp. n. [(26) vaginal palpi, (27) spiculum ventrale], (28, 29) P. submontanus sp. n. [(28) spermatheca, (29) vaginal palpi].

fers from it only in the structure of the aedeagus: aedeagus uniformly weakly widened from base nearly to apex, then sharply narrowed toward apex, less strongly and not regularly curved in lateral view, with more straight basal $2 / 3$ and with lower surface nearly flat in second basal $1 / 4$ and then nearly sharply concave. In addition, the anterolateral callosity of the pronotum of the new species more strongly project beyond the contour. P. laxus differs from the closely related $P$. punctifrons Baly in the following characters: shagreenity of body stronger, vertex flatter, punctures on vertex less clearly visible against background of shagreenity, 1st segment of fore tarsus of male larger, strial punctures of elytra smaller and frequently elongate, secondary punctation of elytra less strongly developed, and hind femur without metallic shine. The- 
ses species also differ in the structure of the male and female genitalia.

This species belongs to the punctifrons speciesgroup.

Distribution. Russia: Khabarovsk Terr., Sakhalin Island.

\section{Psylliodes submontanus Nadein, sp. n.}

Material. Holotype: ô, "Russia, Stavropol Terr., Pyatigorsk, Mashuk Mt., botanical station environs Kar'er, 27.VII.2003 M. Volkovitsh leg." Paratypes: 1

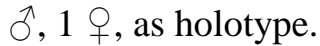

Description. Body oblong-oval, moderately convex, dorsally dark, with weak bronze shine; shine of pronotum more intensive than that of elytra; elytra occasionally without bronze shine; head with red appearing through, paler, with brighter shine; antennae, fore and middle legs, and hind tibia fuscous-yellow; hind femur brownish in middle and upper parts; ventrally body dark brown to black.

Head not large; eyes medium-sized, convex. Vertex weakly convex, punctate between eyes; punctures fine, slightly smaller than those on pronotum, slightly irregular-shaped, superficial, arranged partly irregularly, occasionally varying in size; intervals between punctures about 0.5-1.0 times puncture diameter, uneven, tubercle-shaped convex, with smoothened shagreenity and short strokes. Ocular sulcus reaching apex of frontal callus, narrow, moderately deep, slightly widened only above upper margin of eye, bearing there several fine setiferous pores; margins of sulcus rather even, not wrinkled. Frontal calli smooth, weakly convex, not separated from vertex, indistinctly separated from frons. Frontal ridge triangular, rather narrow, moderately convex, flattened in middle, with smoothened shagreenity; anterofrontal ridge weakly convex, narrow. Antennal groove superficial, with coarse shagreenity. Labrum narrow and long; only two inner setiferous pores well developed, large, and deep.

Pronotum moderately convex, with slightly rounded sides distinctly converging forwards; edging of anterior margin very narrow, not convex, that of posterior margin distinct, wider, and convex, edgings of lateral margins narrow and smooth; anterolateral callosity well developed, moderately thickened, distinctly projecting beyond contour, especially near setiferous pore, forming there distinct angle; punctures on pronotum fine, as large as, or slightly larger than those on vertex, but smaller than those in striae of elytra, super- ficial, occasionally varying in size and shape; intervals between punctures $0.5-1.5$ times puncture diameter; intervals between punctures not very even, but not convex, with smoothened and rather indistinct shagreenity.

Scutellum triangular, convex, shagreened, with sharp apex. Elytra moderately long and moderately convex; humeral calli poorly developed; hind wings reduced; punctures in elytral striae large, deep, distinctly smaller and shallower at apex than at base; striae slightly depressed, intervals between them slightly convex; intervals between punctures about half puncture diameter, intervals between striae equally $2.0-2.5$ puncture diameter; secondary punctation consisting of fine superficial punctures arranged moderately densely, punctures occasionally forming 1-2 confused striae; intervals between punctures slightly uneven, with very fine, smoothened, indistinct shagreenity. Epipleura convex, shagreened, and even in basal half, becoming pleated-wrinkled and tuberculate in apical half.

Hind tibia moderately long, distinctly but not strongly curved, weakly widened in apical $1 / 3$ in dorsal view; inner ridge with large tooth (Fig. 13). First segment of hind tarsus straight. First segment of fore tarsus of male distinctly larger than that of female, but moderately wide (Fig. 5). Structure of tegmen similar to typical one (Fig. 8). Aedeagus as in Fig. 19.

Female. Spermatheca with narrow and long collo, nodulus moderately narrow, ductus long and curved (Fig. 28). Pygidium as in Fig. 31, vaginal palpi as in Fig. 29, speculum ventrale as in Fig. 30.

Length of body of holotype $2.52 \mathrm{~mm}$, width 1.26 $\mathrm{mm}$; those of paratypes $2.59-2.72 \mathrm{~mm}$ and $1.32-1.4$ $\mathrm{mm}$, respectively; PI 1.47-1.56 (1.51), EI 1.42-1.60 (1.52), LI 3.30-3.41 (3.37), BI 1.85-2.06 (1.97), WI 1.41-1.59 (1.47).

Differential diagnosis. The new species is closely related to $P$. napi (F.) in the structure of the aedeagus and clearly differs from it in the coloration of the body, shape of the hind tibia, structure of the head and spermatheca, and in a number of other characters, including the structure of the tegmen. The new species is preliminary included in the napi species-group, but it is also similar to the apterous P. ozisiki Leon et Arnold from the cupreus species-group; the latter species is known to me only from the literature (Leonardi and Arnold, 1995).

Distribution. Russia: Stavropol Terr. 

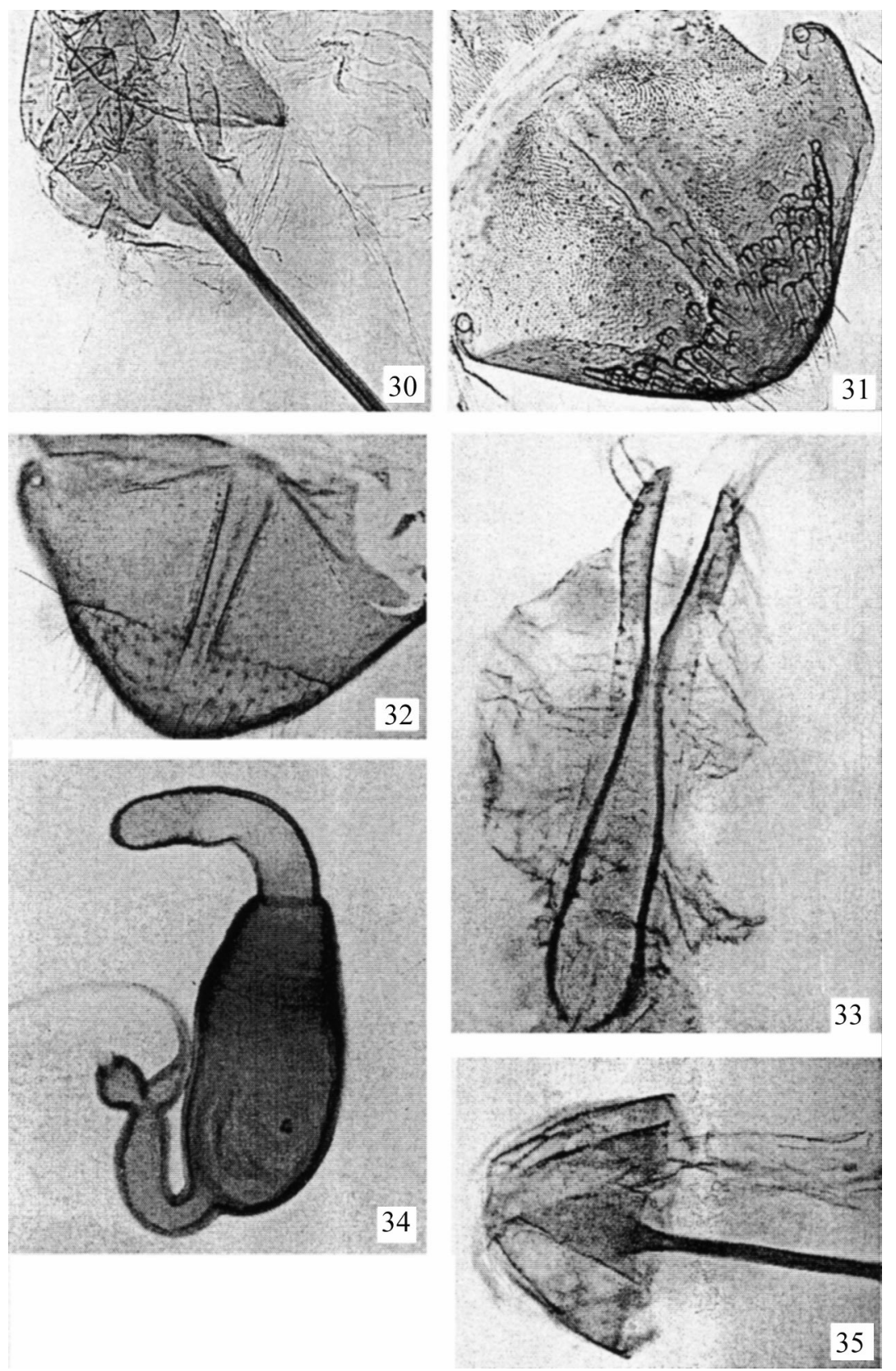

Figs. 30-35. Psylliodes Latr.: (30, 31) P. submontanus sp. n. [(30) spiculum ventrale, (31) pygidium], (32-35) P. concolor sp. n. [(32) pygidium, (33) vaginal palpi, (34) spermatheca, (35) spiculum ventrale].

Psylliodes concolor Nadein, sp. n.

Material. Holotype: đo, "Manglis, 1880," "k. G. Siversa [in Cyrillic]." Paratype: "Martkobi 16/17, Juni 1879," "k. G. Siversa [in Cyrillic]," ㅇ.

Description. Body oblong-oval, weakly convex, entirely fuscous-yellow, only pronotum and body ventrally occasionally slightly darker and elytral suture weakly and very narrowly darkened.

Head small; eyes small, weakly convex. Vertex nearly flat, wide, uneven, covered with rather fine, superficial, hardly visible punctures varying in size and arranged irregularly, mainly sparsely. Average length of intervals between punctures about twice puncture diameter, intervals more or less smooth, but not quite even. Ocular sulcus uneven, moderately deep and wide, adjoining eye only along its upper margin, then separated by convex interval gradually becoming wider; sulcus reaching upper margin of frontal calli, turning there into supracallinal sulcus; margins of sulcus uneven, bottom and margins with several setiferous pores. Frontal calli rather wide, moderately con- 
vex, separated from vertex by rather fine and indistinct line, also not very distinctly separated from frons; lateral margins of calli merging with convex area in front of inner margin of eye; calli separated by superficial depression. Frontal ridge widely triangular, weakly projecting above surface of frons, flattened on upper side; anterofrontal ridge moderately convex and wide, as smooth as frontal ridge. Antennal sockets widely spaced, distance from socket to inner margin of eye not exceeding half diameter of socket. Antennal groove superficial, with smoothened shagreenity. Labrum wide, not long; all setiferous pores equally developed.

Pronotum rather large, wide, only slightly narrower than elytral base, moderately convex; sides distinctly converging forwards, rather weakly rounded; edging of anterior margin very fine and hardly visible, that of posterior margin wider, convex; edgings of lateral margins narrow and smooth; basal margin with 2 rather short superficial strokes resembling punctureshaped depression; anterolateral callosity small and not thickened, weakly projecting beyond contour mainly in area of setiferous pore, forming there distinct rounded angle; punctation dense, average length of intervals between punctures approximately equal to puncture diameter; punctures rather fine, larger than those on vertex, but significantly smaller than those in elytral striae, superficial; intervals between them nearly flat, with fine, smoothened, indistinct shagreenity.

Scutellum small, convex, very finely shagreened, with slightly obtused apex. Elytra elongate, moderately convex, wide; lateral margins uniformly weakly rounded; punctures in striae medium-sized, nearly as large at elytral base as at apex, moderately deep, regular-shaped, very dense; intervals between punctures not greater than half puncture diameter; striae depressed to form sulci; intervals between them weakly convex, 1.5-2.0 times puncture diameter; secondary punctation fine, superficial, moderately dense; punctures trending to form 1-2 confused striae; intervals between punctures moderately smooth. Epipleura narrow at base, with fine pleat-wrinkled and tuberculate sculpture along nearly entire length. Elytral apices separately weakly rounded toward suture; sutural angle distinct, obtuse.

Hind tibia saber-shaped, moderately wide, wide in dorsal view, inner and outer ridges without teeth; apical $1 / 3$ with not very dense, rather fine spines dorsally
(Fig. 12). First segment of hind tarsus weakly curved. First segment of fore tarsus of male slightly larger than that of female, rather narrow and rather short (Fig. 4). Structure of tegmen similar to typical one (Fig. 7). Aedeagus as in Fig. 18.

Female. Spermatheca with moderately long and wide collo, nodulus rather narrow, ductus rather short and wide, not forming loops, ramus large (Fig. 34). Pygidium as in Fig. 32, vaginal palpi as in Fig. 33, spiculum ventrale as in Fig. 35.

Length of body of holotype $2.27 \mathrm{~mm}$, width 1.34 $\mathrm{mm}$; those of paratype $2.94 \mathrm{~mm}$ and $1.54 \mathrm{~mm}$, respectively; PI 1.46-1.47 (1.47), EI 1.45-1.56 (1.51), LI 3.13-3.20 (3.16), BI 1.91-2.06 (1.99), WI 1.37-1.49 (1.43).

Differential diagnosis. The new species is most closely related to P. 1uteolus (Mull.) and differs from it in the less convex vertex, smaller eyes, mainly denser punctation of the pronotum and elytral striae, more strongly depressed elytral striae, smaller spines on apical $1 / 3$ of the hind tibia, larger size, and structure of the genitalia of both sexes. P. concolor differs from $P$. wachsmanni Csiki in the structure of the aedeagus (Leonardi, 1972). According to the characters, this species belongs to the luteolus species-group (Nadein, 2006).

\section{Distribution. Georgia.}

Psylliodes diversicolor Nadein, sp. $\mathrm{n}$.

Material. Holotype: $\widehat{\delta}$, "Turkey, Karaman, $8.5 \mathrm{~km}$ E Ayranci, 1177 m, 27.VI.2005 M. Volkovitsh leg." Paratypes: $1 \hat{\jmath}$, as holotype; $2 \hat{\jmath}$, "Turkey, Antalya, $4.5 \mathrm{~km}$ NW Akseki, 1325 m, 26.VI.2005 M. Volkovitsh leg."

Description. Body widely oval, weakly convex; head and prothorax rufous-red; elytra, meso- and metathorax, and abdomen dark brown; legs, except for brownish hind femur, and antennae fuscous-yellow.

Head small; eyes not very large, moderately or weakly convex. Vertex weakly convex, wide, with indistinct punctation; punctures fine, superficial, rather sparse, hardly visible; intervals between them occasionally not quite even, with smooth or smoothened shagreenity. Ocular sulcus short, wide, superficial, distinct, with even margins, adjoining eye only above upper margin of eye, then separated from it by wide interval and reaching frontal calli, turning there into 
supracallinal sulcus; several small setiferous pores, forming no depressions, lying near outer margin of sulcus. Frontal calli moderately wide, almost convex, separated from vertex and frons by fine, hardly visible lines; margins of calli merging with convex area in front of inner margin of eyes, surface of calli nearly smooth. Frontal ridge short, wide, trapeziform, rather weakly convex, flattened, nearly smooth; anterofrontal ridge narrow, nearly convex. Antennal groove narrow, almost not concave, shagreened. Labrum long, not wide; all 6 setiferous pores developed, 2 inner pores largest and deepest.

Pronotum wide, moderately convex, only slightly narrower than elytral base; lateral margins distinctly converging forwards, rounded, arcuately upcurved in lateral view; basal margin with 2 small short wide superficial strokes. Edging of anterior margin fine and flat, that of posterior margin slightly wider and more convex; edgings of lateral margins narrow and smooth; anterolateral callosity poorly developed, not thickened, weakly projecting beyond contour; punctation distinct, punctures small, significantly smaller than those in striae of elytra, superficial; intervals between punctures subequal, on average, equal to puncture diameter, flat, nearly smooth or with smoothened shagreenity.

Elytra elongate, moderately convex, weakly rounded at sides; humeral calli small and weakly convex. Punctures in striae large, forming more or less depressed sulci; intervals between striae more or less convex; distance between punctures in striae about half puncture diameter; intervals between striae equal to 1-2 puncture diameters, more frequently, 1.5 times puncture diameter; punctures in striae slightly smaller at apex than at base; secondary punctation almost not pronounced to moderately coarse and dense (in large individuals); intervals between punctures nearly smooth. Elytral apices very weakly rounded toward suture, sutural angle nearly rectangular, distinct.

Hind tibia short, wide in dorsal view, saber-shaped in lateral view, not very strongly curved, moderately wide; apical $1 / 3$ of tibia almost not widened, with rather fine and not very dense spines (Fig. 9). Fore tibia of male short and thickened. First segment of hind tarsus short and moderately wide, straight. First segment of fore tarsus of male large and wide (Fig. 1). Structure of tegmen occupying intermediate position between typical and modified ones (Fig. 6). Aedeagus as in Fig. 15.

Length of body of holotype $2.9 \mathrm{~mm}$, width $1.32 \mathrm{~mm}$; those of paratypes $2.1-2.65 \mathrm{~mm}$ and 0.98
$1.34 \mathrm{~mm}$, respectively; PI 1.46-1.59 (1.53), EI 1.511.65 (1.59), LI 2.98-3.30 (3.14), BI 1.97-2.20 (2.09), WI 1.24-1.37 (1.30).

Differential diagnosis. According to a set of the characters, the new species belongs to the luteolus species-group (Nadein, 2006), but differs from the all species of this group in the bicolor body and in the structure of the aedeagus. Such a coloration was found in P. picinus ab. rufopiceus Letzner belonging to the closely related picinus species-group, also known from Turkey (Warchalowski, 2003). However, the P. diversicolor clearly differs from this species in many characters. The new species clearly differs from $P$. ruficolor Doguet and P. pallidicolor Pic in the significantly wider 1 st segment of the fore tarsus of the male, thicker fore tibia, wider head with the less convex vertex and frontal calli, wider (wider than long) pronotum with the rounded lateral margins, denser punctures in the elytral striae, less strongly curved and narrower hind tibia, and wider 1st segment of the hind tarsus; the species additionally differs from $P$. luteolus in the smaller eyes and wider vertex and pronotum and in the structure of the hind tibia.

Distribution. Turkey.

Psylliodes insularis Nadein, sp. $\mathrm{n}$.

Material. Holotype: + , "Canaries," "Psylliodes marcida Illig."

Description. Body oblong-oval, elongate, entirely rufescent-yellow, with weak metallic shine; legs and antennae yellow.

Head small; eyes medium-sized, moderately convex. Antenna rather short, with rather slender segments. Vertex rather wide, nearly flat, densely punctate between eyes; punctures large, deep, as large, or nearly as large as those on pronotum; intervals between punctures not exceeding their diameter, frequently shorter than puncture diameter, convex, uneven, coarsely and distinctly shagreened. Ocular sulcus wide, deep, adjoining margin of eye along nearly entire length, separated from margin of eye only near frontal callus, reaching antennal socket; margin of sulcus from side of vertex indistinct, flat; sulcus widened at upper inner margin of eye owing to several setiferous pores forming depression. Frontal calli narrow, almost flat, shagreened and punctate, separated from vertex by superficial lines. Frontal ridge narrow, triangular, convex, shagreened. Surface of frons below antennal sockets rather strongly depressed. Antennal 
groove moderately deep, coarsely shagreened, tuberculate. Labrum rather small, short, moderately wide. Two inner setiferous pores significantly larger and deeper than others.

Pronotum medium-sized, wider than long; lateral margins distinctly converging forwards; edging of anterior margin very fine, nearly flat; that of posterior margin thicker and more convex; edgings of lateral margins narrow and smooth. Anterolateral callosity large, thickened, weakly projecting beyond lateral contour, strongly rounded near setiferous pore, not forming angle. Punctation dense, mostly regular; punctures not very distinct and not deep, average length of intervals between them equal to puncture diameter; intervals uneven, weakly convex on disc and more strongly convex on lateral declivities, distinctly and coarsely shagreened.

Elytra elongate, very weakly regularly rounded at sides. Humeral calli not developed, hind wings absent. Striae forming rather superficial sulci separated by flat intervals; punctures in striae fine, only slightly larger than those on pronotum, not very distinct and deep, smaller at apex than at base; average length of intervals between punctures in striae equal to puncture diameter; intervals between striae 2.0-3.5 times puncture diameters, with tendency toward formation of dual striae. Secondary punctation well developed; punctures large, moderately deep, forming 1-2 confused rows. Intervals between punctures on disc with smoothened shagreenity, uneven, more distinctly shagreened at margins. Elytral apices separately not very strongly rounded toward suture, sutural angle rounded, not pronounced.

Hind tibia rather narrow, nearly straight, widened in apical $1 / 3$ only in area of tarsal joint (Fig. 14). First segment of hind tarsus narrow, straight.

Spermatheca with narrow and long collo; nodulus moderately narrow; ductus short, narrow, not forming loops (Fig. 22). Pygidium as in Fig. 21, vaginal palpi as in Fig. 20, spiculum ventrale as in Fig. 23.
Length of body $3.65 \mathrm{~mm}$, width $1.65 \mathrm{~mm}$; PI 1.60 , EI 1.74, LI 3.73, BI 2.21, WI 1.34.

Differential diagnosis. The new species belongs to the chrysocephalus species-group (Nadein, 2006) and is closely related to $P$. chrysocephalus (L). The yellow coloration of the body rather rarely occurs in numerous forms and variations of $P$. chrysocephalus. This coloration is known for the v. sordida Steph., f. kunzei Foudr., and f. anglica F. variations. The new species differs from the listed and other forms in the less convex vertex, small eyes, larger punctures on the vertex, distinct and coarser shagreenity of the vertex, less convex and longer pronotum, finer punctation of the elytra, more slender hind tibia, and absence of wings. $P$. insularis also differs from f. anglica in the yellow pronotum. $P$. chrysocephalus is known from Madeira, Cabo Verde, and the Azores, being presented there by a form with the typical coloration.

Distribution. Canary Islands.

\section{ACKNOWLEDGMENTS}

The author is grateful to M.G. Volkovitsh for the material supplied for examination.

\section{REFERENCES}

1. Leonardi, C., "La "Psylliodes wachsmanni" Csiki specie distinta e suo inquadramento nel gruppo della "Psylliodes picina" (Coleoptera, Chrysomelidae)," Atti Mus. Civ. Stor. Natur. Trieste 28 (1), 137-146 (1972).

2. Leonardi, C. and Arnold, U., "Due nuove specie di Psylliodes della regione mediterranea orientale (Coleoptera, Chrysomelidae)," Atti Mus. Civ. Stor. Natur. Milano 134 (1993), 299-311 (1995).

3. Nadein, K., "A Significance of the Tegmen Structure for the Classification of the Genus Psylliodes Latrielle, 1829 (Coleoptera, Chrysomelidae: Psylliodina), Trudy Russ. Entomol. O-va 77, 250-254 (2006).

4. Warchalowski, A., Chrysomelidae. The Leaf-beetles of Europe and Mediterranean Area (Nature optima dux Foundation, Warszawa, 2003). 